\title{
Properties of Orthodontic Clear Aligner Materials - A Review
}

\author{
Binu Punnoose Gold ${ }^{1}$, Suvetha Siva ${ }^{2}$, Sangeetha Duraisamy ${ }^{3}$, Azmina Idaayath ${ }^{4}$, Ravi Kannan ${ }^{5}$ \\ 1, 2, 3, 4, 5 Department of Orthodontics, SRM Dental College, Ramapuram, Chennai, Tamil Nadu, India.
}

\section{ABSTRACT}

\section{BACKGROUND}

Clear aligners are orthodontic devices that are transparent, a plastic used to correct malaligned teeth. Here patient wears a series of customized clear, removable aligners that gradually move the teeth to the desired position. The clear aligner system is a modern adaptation of the systems described since the middle of the 20th century, therefore there were different devices and philosophies that have led to its creation and the system has evolved a lot over the decades.

Clear aligner therapy has been a part of the orthodontic practice for years, but, popularity was increased since the introduction of Invisalign appliances (Align Technology) in 1998. There are almost 27 different clear aligner products currently on offer for orthodontic treatment. Nowadays, more people prefer clear aligner treatment because it is aesthetically superior to brackets and lingual orthodontics. The superiority of clear aligners lies in their aesthetics. The optical properties of the clear aligner material play a major role in aesthetics. The rising demand among adult patients for "invisible" orthodontic treatment has led to an exponential growth in the clear aligner market.

Indeed, these aligners have a low aesthetic impact, as well as being able to effectively and progressively guide the teeth into their programmed positions. They are also removable and therefore do not hamper oral hygiene maintenance, in turn reducing the risk of white spots, caries, gingivitis and periodontal disease. All the materials do not possess the same chemical composition. The properties change before and after wear. In this article, we bring out the different materials used for the manufacture of clear aligners and their various properties.

\section{KEY WORDS}

Clear Aligners, Optical Properties, Thermoplastic Material, Mechanical Properties.
Corresponding Author:

Dr. Binu Punnoose Gold, 216, Gandhi Nagar, 2nd Street,

Vazhuthacaud, Thycaud P.O

Thiruvananthapuram - 695014,

Kerala, India.

E-mail: binupgold@gmail.com

DOI: $10.14260 / \mathrm{jemds} / 2021 / 668$

How to Cite This Article:

Gold BP, Siva S, Duraisamy $S$, et al. Properties of orthodontic clear aligner materials - a review. J Evolution Med Dent Sci 2021;10(37):3288-3294, DOI: 10.14260/jemds/2021/668

Submission 12-06-2021, Peer Review 05-08-2021, Acceptance 12-08-2021, Published 13-09-2021.

Copyright (c) 2021 Binu Punnoose Gold et al. This is an open access article distributed under Creative Commons Attribution License [Attribution 4.0 International (CC BY 4.0)] 


\section{BACKGROUND}

Clear aligner therapy is an orthodontic treatment modality in which the patient wears a series of customized clear, removable aligners that gradually move the teeth to the desired position. The total number of aligners varies depending on the severity of the malocclusion. ${ }^{1}$ The superiority of clear aligners lies in their invisibility which is attributed to their optical properties. ${ }^{2}$

Adult patients select clear aligner treatment despite the escalated cost and orthodontists practice clear aligners despite its biomechanical limitations because of the aesthetics it offers. The optical properties of the different clear aligners vary because of the composition of the materials used in manufacturing. 3,4 The various materials used by different manufacturers for the fabrication of orthodontic clear aligners are polyurethane, polyvinyl chloride(PVC), polyethylene terephthalate glycol (PET - G).

Large spring back, high stored energy, tolerance to the oral environment, biocompatibility, and low surface roughness would be ideal material features for a transparent aligner. The mechanical properties of the aligner's thermoplastic material are critical in providing continuous orthodontic forces capable of producing the required results. Transparency, which is determined by optical properties, lower hardness, improved elasticity and resilience and ageing resistance are all desirable qualities in orthodontic thermoplastic materials. ${ }^{5}$ Polyethylene copolymers and polypropylene polymers were the two most prevalent materials utilized for vacuum produced retainers at first. When bite planes need to be incorporated into the device, polyethylene polymers are the plastic of choice since they allow the acrylic to be attached to the material. Because it is nearly transparent, the material is also seen to be more attractive. 6 Polypropylene polymers are more robust and flexible than polyethylene polymers, but they are less attractive since the substance is translucent. ${ }^{7}$ Polyurethane material was introduced in 2013, which is currently considered the best for aligner therapy. This is because of the high strength and high toughness it offers. ${ }^{8}$

It is critical to understand the many clear aligner products on the market and how they differ in terms of construction material, thickness, and the clinical protocol to acquire the best treatment results. ${ }^{8}$ The purpose of this review article is to highlight the characteristics of the materials used by various companies to make orthodontic clear aligners.

\section{HISTORY OF CLEAR ALIGNERS}

When Kesling pioneered the notion of utilising a succession of thermoplastic tooth positioners to progressively reposition malaligned teeth to improved positions in 1946, the prospect of using clear orthodontic appliances was established. ${ }^{9} \mathrm{He}$ used a material that was similar to clear thermoplastic rubber for the fabrication of the same. Ponitz (1971) developed an "Invisible Retainer" based on Kesling's concept of pre-positioning teeth on a master study model. The 'Invisible Retainer,' like Kesling's appliance, could only cause tiny tooth movements, achieving its results by tipping crowns. Biocryl (polymethylmethacrylate) was the substance utilised. ${ }^{10}$

The idea for orthodontic aligners came from Dentsply Raintree Essix plastic, which was used to support anterior teeth. Jack Sheridan coined the word Essix to designate his aesthetic method for straightening the six anterior teeth more than three decades ago. In 1993, Sheridan described a procedure that combined clear aligners with interproximal teeth reduction. The material used for Essix was polypropelene. ${ }^{11}$

The clear aligner treatment (CAT) was introduced in 1997 by Align Technology (Santa Clara, Calif.), which modified and incorporated modern technologies to make Kesling's notion a viable orthodontic treatment option. Align technology brought this treatment into the lab, allowing doctors to subcontract it. Invisalign was introduced by Align Technology in 1999.12 It was the first orthodontic device to use the computer-aided design (CAD) and manufacture (CAM). This method allows various tooth setups to be generated from a single impression, rather than requiring a new impression for each tooth movement.

The most popular clear aligner system is Align Technology's Invisalign, which consists of a set of digitally manufactured thermoplastic aligners created using stereolithographic models. Removability for ease of eating, comfort, and oral hygiene maintenance, enhanced aesthetics, and reduced periodontal risk are the key benefits of CAT. Furthermore, because it is smaller than standard orthodontic appliances, it has enhanced patient acceptability of orthodontic therapy. ${ }^{13}$ The material used in invisalign is smart track multilayer polyurethane and copolyester proprietary material.

\section{Types of Clear Aligners}

Clear aligners can be categorized into four basic types.

- Type 1: Commonly known as positioners or guides which include Kesling's tooth positioner.

- Type 2: Thermoformed plastics like Essix retainers, spring aligners.

- $\quad$ Type 3: Aligners that are made from models cut out to correct position to align teeth when done in a series of models. They produce minor tooth movement using four or five aligners.

- $\quad$ Type 4: Aligners fabricated using digital technology that can treat minor to complex malocclusions including posterior and anterior segments. There is increased precise tooth movement with every change in the aligner.

\section{Indications for Aligner Therapy}

Joffe suggested that the clear aligner is most successful for treating the following problems

- Malocclusion is caused by mild crowding and misalignment (1 $-5 \mathrm{~mm})$.

- Treatment that involves minor lateral and / or anteroposterior expansion, minor interproximal tooth reduction, or the extraction of a lower incisor.

- $\quad$ Spacing issues (between 1 and $5 \mathrm{~mm}$ ). 
- In deep overbite cases (Class II division 2 type malocclusions) where the overbite can be decreased by intrusion and advancement of incisors.

- Narrow arches where expansion can be done without tipping the teeth too much.

\section{Advantages of Clear Aligners}

Aligners are preferred over traditional fixed appliances for a variety of reasons.

- They are clear and hence more aesthetically appealing, especially for adults.

- They are simple to remove, allowing for better dental hygiene.

- Aligners are more comfortable to wear in general.

- The advantages of periodontal health benefits may also be associated with clear aligner treatment.

\section{Materials Used in Clear Aligners}

According to the literature and catalogues of other clear aligner firms, PET - G is currently utilized by the majority of other aligner producers, but polypropylene, polycarbonate (PC), thermoplastic polyurethanes (TPU), ethylene-vinyl acetate and a variety of other materials are also used. In 2013 , polyurethane material was introduced, and it is now widely regarded as the best material for aligner therapy.

Clear aligners are available in a variety of thicknesses, ranging from $0.50 \mathrm{~mm}$ to $1.5 \mathrm{~mm}$. This, like the building material, can have an impact on their mechanical qualities and, as a result, their performance. ${ }^{14}$

The colour stability and transparency of orthodontic clear aligners should be stable during the 2 - week orthodontic treatment period from an aesthetic standpoint. ${ }^{15}$ Various elements, like ultraviolet irradiation, staining beverages, and mouthwashes, might often affect the colour stability of dental materials. According to studies, pigment absorption from food and drink in the mouth cavity is an issue for PU - based elastomeric ligatures. Patients are frequently advised to take out the clear aligners when they eat or drink anything other than water. The pigments in staining agents may build-up in patients who do not follow the directions, causing colour changes in the aligner materials. As a result, even throughout the 2 - week's treatment, clear aligners may become less cosmetically pleasing, which is a clinical concern. The appearance of orthodontic aligners has the potential to affect societal attitudes, and hence plays a role in patients' decision to use them. Clear aligner functionality in terms of mechanical qualities and force delivery features, on the other hand, is critical for orthodontic treatment.

\section{Thermal Properties}

Thermoplastic materials are linear or slightly branched polymers with strong intramolecular covalent bonds and weak intermolecular Van der waal's forces. The bonds melt and the polymers overflow at raised temperatures and the molecular chains solidify into new shapes when cooled. The materials do not undergo chemical changes at altered temperatures, hence the process of softening with heating and hardening with cooling may be repeated. In solid-state, the thermoplastic materials used for making clear aligners are semi-crystalline with amorphous strands of polymers dispersed in between. The crystalline polymers are more transparent with well-defined melting points and glass transition temperature. ${ }^{13}$

\section{Mechanical Properties}

Aligners are subjected to both short-term and long-term loading forces in the oral cavity. When the aligner is fitted to the dentition, the aligner material is loaded with short term forces, after the fit in. When the aligner is worn by the patient it experiences long term forces due to the displacement caused by programmed tooth movement and the biomechanical forces generated from the musculoskeletal system. ${ }^{16}$

The clear aligners decrease the surface roughness after one week of use and it further decreases after wearing for subsequent weeks. Roughness parameters and mechanical properties deterioration have an undesirable effect on invisalign appliance retention and orthodontic pressures during orthodontic treatment. ${ }^{17}$

The thermoforming procedure used to make clear aligners was very repeatable, and intraoral ageing revealed no clinically significant alterations that could influence the efficacy of the orthodontic aligners. ${ }^{18}$

Thermoplastic materials follow simple Hook's law for forces of short duration. Within the elastic limit of the curve, the stress is proportional to strain, the material exhibits an elastic behaviour and returns to its original size and shape when stress is removed (Fig. 1).

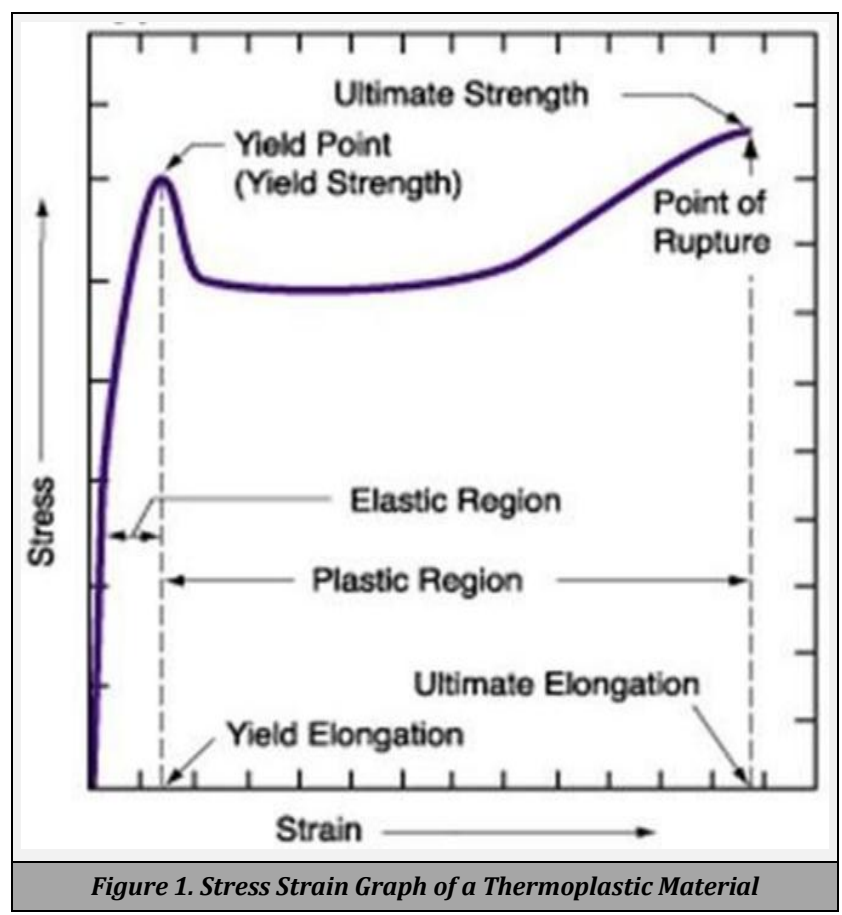

The yield strength is the stress beyond which the strain is no longer proportional to stress and the material is no longer elastic and behaves like plastic. The ultimate tensile strength is the stress at which the material ruptures and toughness is the energy absorbed by the material before it ruptures and is calculated by calculating the area under the stress strain curve. Thermoplastics with higher yield strength, ultimate 
tensile strength and toughness are desirable for the manufacturing of clear aligners.

Young's modulus or the modulus of elasticity of the material is the ratio between stress and corresponding strain. The young's modulus is a unique character of the material and is a measure of the material stiffness. For a clear aligner, the material should possess sufficient stiffness to generate the forces and moments required for achieving the programmed tooth movement.

If a material with a very high modulus is used for the fabrication of the aligner, the aligner would be very stiff and the patient will experience difficulty in insertion and removal of the appliance. Similarly, a material with low stiffness will not generate enough force to move teeth.

When thermoplastic aligners are subjected to long term forces they do not follow Hooks law, they exhibit viscoelastic properties showing a time-dependent relationship between the stress and strain which has a profound influence on the performance of the aligner. When the aligner is fitted in the arch, the elastic nature of the material allows the aligner to deform and stretch and generate forces required for the programmed tooth movement.

As time lapses, due to the viscoelastic nature of the material, the force generated by the appliance for the same deformation decreases. This stress relaxation process is affected by the material, the temperature of the oral environment and the severity of the load to which the particular part of the aligner is subjected. The type and thickness of the material used in fabrication affect the elastic properties of the aligners to a greater extent.

When compared to uncured 3D printed aligners and thermoformed aligners, cured 3D printed aligners had greater dimensional accuracy and compressive mechanical strength. Dental LT resin, which is used to make clear dental aligners, is a biocompatible substance with an acrylic ester monomer. 15

The forces that aligners apply must be within the biological limitations of what human teeth can tolerate, which is around $0.0050 \mathrm{MPa}$. These stress values can also help with sheet thickness selection for aligners. Finally, apart from comfort, the efficiency of every new aligner for a patient must be determined by analysing applied stress. Polyether compounds are tougher than polyester urethanes and have greater moisture resistance and dynamic characteristics. They also perform better at low temperatures. Polyether materials, on the other hand, have the lower abrasion resistance and are more likely to tear. ${ }^{18}$

\begin{tabular}{|c|cc|}
\hline Properties & $\begin{array}{r}\text { Polyester Based } \\
\text { Thermoplas } \\
\text { tic Urethane }\end{array}$ & $\begin{array}{r}\text { Polyether-Based } \\
\text { Thermoplasti } \\
\text { c Urethane }\end{array}$ \\
\hline Abrasion resistance & Excellent & Good \\
Chemical resistance & Excellent & Good \\
Microbial resistance & Very poor & poor \\
Low temperature flexibility & Acceptable & Excellent \\
Adhesion to polar substrates & Good & Acceptable \\
\hline Table1. Mechanical Properties of Thermoplastic Polyurethane \\
\hline
\end{tabular}

\section{Chemical Resistance}

The aligners are constantly exposed to saliva at a constant body temperature which can adversely affect the chemical composition of polymers. Certain polyesters like, polycarbonate and polyamides may undergo irreversible hydrolysis that may degrade the polymer structure. The polymer used for aligner fabrication should be resistant to hydrolysis and water degradation.

\section{Optical Properties}

Aligner materials should exhibit good light transmittance and should be able to transmit $80 \%$ of the visible light. Amorphous polymers exhibit high translucency and are preferred as aligner materials over crystalline polymers which are highly opaque and unaesthetic. Polyurethane, polyester, polyvinylchloride, polysulfone and polycarbonate are some of the polymer materials that exhibit good optical properties suitable for aligner fabrication. ${ }^{2}$

The colour change values of the polyurethane-based Invisalign aligners were much higher than those of the polycarboxylate and polyethylene terephthalate glycol-based aligners. Polyurethane is also susceptible to pigment adsorption and does not provide enough colour stability, according to the literature.3,4 Aligners should be removed before eating and drinking during treatment. ${ }^{2}$ The clear aligner was likewise rated as more attractive. According to these findings, a lower level of appliance display is required for societal acceptance.

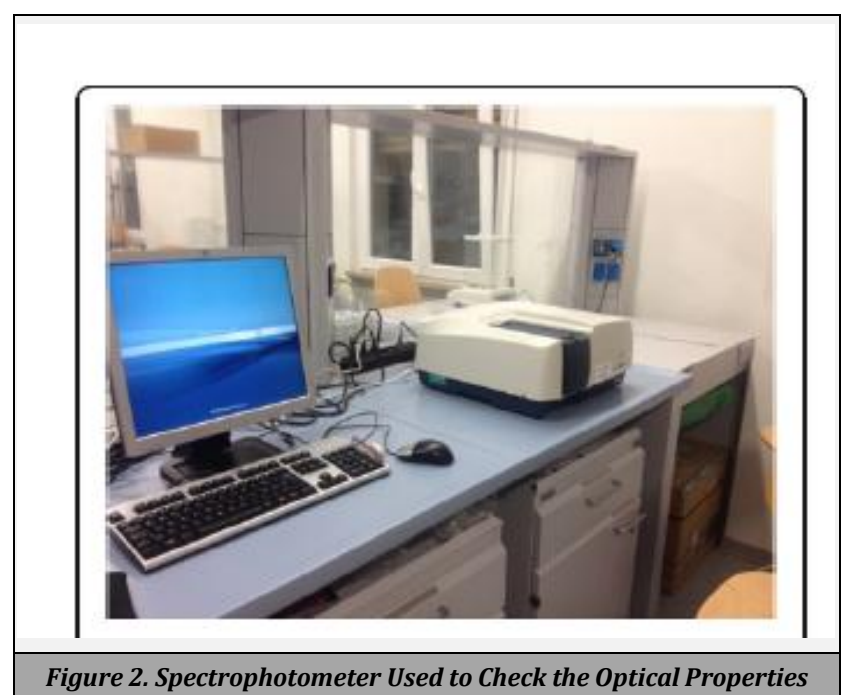

\section{Material Adaptation}

The ability of the material to adapt or conform to the models during the thermoforming process is an important requirement of an aligner material. At a defined temperature around $110^{\circ} \mathrm{C}$, the level of polyurethane material is superior to that of other materials. ${ }^{3,4}$

\section{Material Stress Cracking}

The resistance to surface cracking under an oral environment when subjected to cyclic stress is of paramount importance to an aligner material. Polyurethane and polycarbonate exhibit higher resistance to surface cracking when compared to other polymers. It is evident from graph 1 that zendura which is made of polyurethane has higher impact strength when compared to other materials. 4 


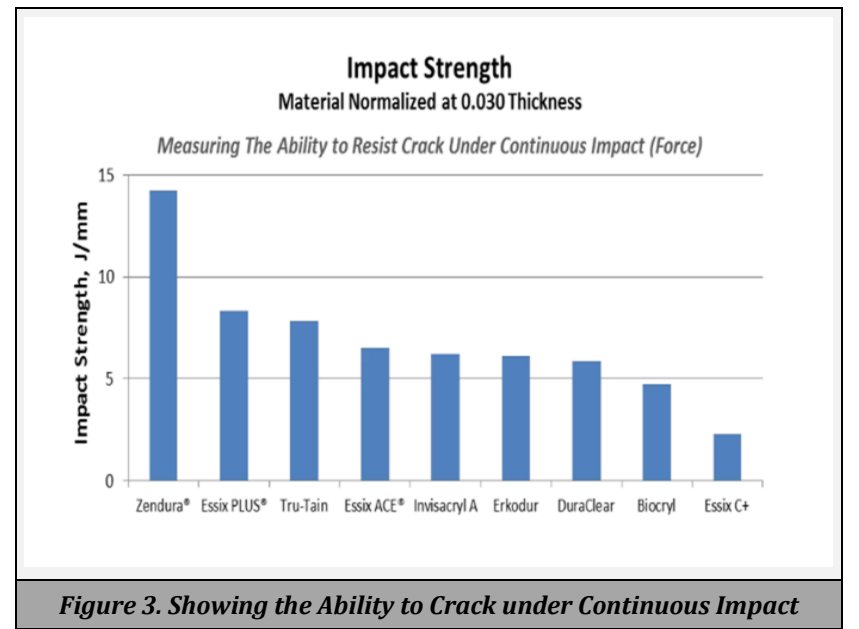

\section{Aligner Durability and Wear Resistance}

The aligner material should be durable under an oral environment and possess high wear resistance to withstand the masticatory forces. If it doesn't, it may lead to the distortion of the material and can lead to deleterious effects.

\section{Biocompatibility}

The polymers should not leach any potential toxins that may produce adverse local or systemic reactions, should not be carcinogenic or produce adverse productive or developmental defects. ${ }^{19}$

\begin{tabular}{|c|c|c|}
\hline Aligner System & Material Used & Manufacturer \\
\hline Duran $®$ & $\begin{array}{l}\text { Polyethylene terephthalate } \\
\text { Glycol }\end{array}$ & $\begin{array}{l}\text { Scheu Dental GmbH (Iserlohn, } \\
\text { Germany), }\end{array}$ \\
\hline Biolon® & $\begin{array}{l}\text { Polyethylene } \\
\text { Terephthalate }\end{array}$ & $\begin{array}{l}\text { Dreve Dentamid GmbH (Unna, } \\
\text { Germany) }\end{array}$ \\
\hline Zendura ${ }^{\circledR}$. & Thermoplastic Urethane & $\begin{array}{l}\text { ZenduraDental (Lakeview Blvd, } \\
\text { Fremont, CA, USA) }\end{array}$ \\
\hline Invisalign & Align Tech. Inc. & $\begin{array}{l}\text { Smart track mult } \\
\text { and copolyester }\end{array}$ \\
\hline & & Zendura \\
\hline MTM clear aligners & Dentsply International & Raintree Es \\
\hline & & Ortolan Pomp \\
\hline Nuvola® & $\begin{array}{c}\text { Polyethylene terephthalate } \\
\text { Glycol }\end{array}$ & $\begin{array}{l}\text { Rhinoceros, Robert McNeel \& } \\
\text { Associates, Rome, Italy) }\end{array}$ \\
\hline \multicolumn{3}{|c|}{ Table 2. Materials Used in Various Clear Aligners ${ }^{1,2,3,4}$} \\
\hline
\end{tabular}

\section{Disadvantages of Clear Aligners}

According to Joffe, there are certain disadvantages of using a clear aligner.

1. Limited control of root movement is observed, particularly for obtaining parallelism of the roots, the correction of important rotations, the recovery axis or the egression. Attachments are essential elements of retention and control of tooth movement.

2. Limited correction of anteroposterior discrepancies: in cases of moderate to severe malocclusion, a surgical phase will be necessary. Until then, the clear aligner treatment phase should be followed by a fixed appliance phase before surgery.

3. The motivation of the patient wearing a removable device cannot be always checked, even after initial acceptance of the system.

4. The lack of control of the operator: once the software is validated, the aligners are delivered in full to the practitioner who will no longer have the opportunity to re-evaluate his treatment plan as is possible in multiattachment. If he encounters significant difficulties preventing him from continuing treatment, a mid-course correction will manufacture new aligners which require a new impression, designing as at the beginning of treatment.

\section{Contraindications for Aligner Therapy}

Conditions that can be difficult to treat with orthodontic clear aligner's altogether are as follows.

- Cases of crowding and spacing where the discrepancy is over $5 \mathrm{~mm}$.

- $\quad$ Skeletal anterior-posterior discrepancies of more than 2 $\mathrm{mm}$ (as measured by discrepancies in cuspid relationships).

- Discrepancies of centric-relation and centric-occlusion.

- Cases where rotation of teeth is severe (more than 20 degrees).

- Open bites cases (both anterior and posterior) that need to be closed.

- $\quad$ Cases where teeth need to be extruded.

- Cases where there is severe tipping of teeth. (More than 45 degrees).

- Teeth where clinical crown length is short.

- Arches where in multiple teeth are missing.

\section{Golden Rules of Invisalign Bio-Mechanics}

Willy Dayan gave golden rules of invisalign bio-mechanics to approach treatment planning with clear aligners.

1. Think like Plastic and Feel like a Tooth: Invisalign is a removable appliance and thus cannot be glued to teeth to "pull" them; the aligner can only "push" on surfaces of the teeth or surfaces of attachments. When a force is placed upon a tooth, it will move according to the biomechanical principles that exist, no matter what the computer screen shows.

2. The ClinCheck Video is not Teeth Moving: The software that shows the virtual arrangement of teeth is not the actual movement of teeth. Think of the images as representing the anatomy of the "changing inner aligner surfaces", and then analyse the resulting forces the aligner will exert upon the teeth.

\section{DISCUSSION}

Due to the rising aesthetic requirement during orthodontic treatment, numerous alternative treatments, like clear aligner therapy, have been developed. With a clear aligner system, a well-thought-out treatment plan is essential. ${ }^{1}$ Clear aligners are particularly advantageous in different ways which include that they are removable, may be custom-made in sequence based on patient-specific malocclusion configurations, and can gradually guide teeth into their programmed positions. All aligners are not made equal, and those currently available vary in terms of construction material, thickness, and therapeutic protocol.2,3

According to the literature and catalogues of other clear aligner firms, PET - G is currently utilized by the majority of 
other aligner producers, but polypropylene, polycarbonate (PC), thermoplastic polyurethanes (TPU), ethylene vinyl acetate, and a variety of other materials are also used.

Clear aligners are available in thicknesses ranging from $0.50 \mathrm{~mm}$ to $1.5 \mathrm{~mm} .{ }^{14}$ This, like the building material, can have an impact on their mechanical qualities and, as a result, their performance.

The colour stability and transparency of orthodontic clear aligners should be stable during the 2 - week orthodontic treatment periods from an aesthetic standpoint. ${ }^{20}$ Various elements, like ultraviolet irradiation, staining beverages, and mouthwashes, might nevertheless, affect the colour stability of dental materials. Pigment adsorption from food and drink in the mouth cavity is a problem for PU - based elastomeric ligatures, according to studies. Patients are frequently advised to take out the clear aligners when they eat or drink anything other than water. The pigments in staining agents may build in patients who do not follow the directions, causing colour changes in the aligner materials. As a result, even throughout the 2 - week's treatment, clear aligners may become less cosmetically pleasing, which is a clinical concern.

Polyurethane material has superiority in the mechanical properties aspect because it offers high strength and toughness. The polymer used for making clear aligners should be resistant to hydrolysis and water degradation.

\section{CONCLUSIONS}

The transparent aligner market has grown at an exponential rate because of the growing demand for "invisible" orthodontic treatment among adult patients.

Understanding both the process of aligner manufacturing and the biomechanics of tooth movement with aligners, as well as how to apply that knowledge to treatment planning and clinical execution, should allow the clinician to design a treatment plan using aligners alone or in combination with fixed appliances to make both simple and complex treatments predictable and routine. Knowing the materials used by various aligner manufacturing companies along with the properties of these materials used help both the clinician and the patient to choose the best aligner available in the market. $\mathrm{Al}$ the properties of the material used to play a pivotal role in the success of the treatment.

Clear aligner treatment ushers in a new era for orthodontists, in which they will no longer be seen as "wire technicians," but rather as cognitive clinicians who must apply their orthodontic skills before beginning active treatment. Future research based on biologic and biomechanical understanding, as well as three-dimensional imaging, is also very promising.

Financial or other competing interests: None.

Disclosure forms provided by the authors are available with the full text of this article at jemds.com.

\section{REFERENCES}

[1] Lombardo L, Arreghini A, Maccarrone R, et al. Optical properties of orthodontic aligners-spectrophotometry analysis of three types before and after aging. Progress in Orthodontics 2015;16:41.

[2] Chen-Lu L, Wen-Tian S, Wen L, et al. Colour stabilities of three types of orthodontic clear aligners exposed to staining agents. International Journal of Oral Science 2016;8(4):246-53.

[3] Zafeiriadis AA, Karamouzos A, Athanasiou AE, et al. An in vivo spectrophotometric evaluation of Vivera and Essix clear thermoplastic retainer discolouration. Australasian Orthodontic Journal 2018;34(1):3-10.

[4] Talic NF, Almudhi AA. The effect of dietary pigmentation on the esthetic appearance of clear orthodontic elastomeric modules. Journal of Orthodontic Science 2016;5(2):70-3.

[5] Barone S, Paoli A, Paolo NE, et al. Mechanical and Geometrical properties assessment of thermoplastic materials for biomedical application. In: Eynard B, Nigrelli V, Oliveri S, et al. eds. Advances on mechanics, design engineering and manufacturing. Lecture notes in mechanical engineering. Springer Cham 2017: p. 437-46.

[6] Ercoli F, Tepedino M, Parziale V, et al. A comparative study of two different clear aligner systems. Progress in Orthodontics 2014;15(1):31.

[7] Kravitz ND, Kusnoto B, BeGole E, et al. How well does Invisalign work? A prospective clinical study evaluating the efficacy of tooth movement withi Invisalign. American Journal of Orthodontics and Dentofacial Orthopedics 2009;135(1):27-35.

[8] Zhang N, Bai Y, Ding X, et al. Preparation and characterization of thermoplastic materials for invisible orthodontics. Dental Materials Journal 2011;30(6):9549.

[9] Kesling HD. The philosophy of the tooth positioning appliance. American Journal of Orthodontics and Oral Surgery 1945;31(6):297-304.

[10] Ponitz RJ. Invisible retainers. American Journal of Orthodontics 1971;59(3):266-72.

[11] Sheridan JJ, LeDoux W, McMinn R. Essix retainers: fabrication and supervision for permanent retention. Journal of Clinical Orthodontics 1993;27(1):37-45.

[12] Bouchez R. Clinical success in Invisalign orthodontic treatment. 1 ${ }^{\text {st }}$ edn. Quintessence Publishing (IL) 2019.

[13] Lombardo L, Martines E, Mazzanti V, et al. Stress relaxation properties of four orthodontic aligner materials: a 24 - hour in vitro study. The Angle Orthodontist 2017;87(1):11-8.

[14] Nahoum H. The vacuum formed dental contour appliance. NY State Dent J 1964;9:385-90.

[15] Rossini G, Parrini S, Castroflorio T, et al. Efficacy of clear aligners in controlling orthodontic tooth movement: a systematic review. The Angle Orthodontist 2015;85(5):881-9.

[16] Fang D, Zhang N, Chen H, et al. Dynamic stress relaxation of orthodontic thermoplastic materials in a simulated oral environment. Dental Materials Journal 2013;32(6):946-51.

[17] Malik OH, McMullin A, Waring DT. Invisible orthodontics part 1: Invisalign. Dental Update 2013;40(3):203-4, 20710, 213-15.

[18] Kohda N, Iijima M, Muguruma T, et al. Effects of mechanical properties of thermoplastic materials on the 
initial force of thermoplastic appliances. The Angle Orthodontist 2013;83(3):476-83.

[19] Iijima M, Kohda N, Kawaguchi $K$, et al. Effects of temperature changes and stress loading on the mechanical and shape memory properties of thermoplastic materials with different glass transition behaviours and crystal structures. European Journal of Orthodontics 2015;37(6):665-70.

[20] Barrer HG. Protecting the integrity of mandibular incisor position through keystoning procedure and spring retainer appliance. Journal of Clinical Orthodontics 1975;9(8):486-94. 